\title{
ESTUDO REFRATOMÉTRICO DE SOLUÇÕES TERNÁRIAS FORMADAS POR ÁGUA, GLICOSE E ACETONITRILA
}

\author{
A.C. Galvão, W.S. Robazza, D. Rodrigues \\ Universidade do Estado de Santa Catarina, Departamento de Engenharia de Alimentos \\ E-mail: alessandro.galvao@udesc.br
}

\begin{abstract}
RESUMO: O estudo de grandezas termodinâmicas de soluções fornecem informações importantes sobre as interações moleculares existentes entre os componentes presentes em uma solução. Estes estudos são fundamentais para o teste, validação e desenvolvimento de teorias de soluções e modelos matemáticos. O estudo refratométrico de uma solução é uma avaliação simples que pode contribuir na compreensão destas interações. Neste contexto, foi estudado o comportamento de soluções binárias de água e glicose, bem como de soluções ternárias de água, glicose e acetonitrila em cinco diferentes temperaturas a partir da determinação do índice de refração da solução. Devido ao enfraquecimento das interações moleculares com o aumento da temperatura, observou-se uma dependência decrescente do índice de refração com a temperatura. A adição de acetonitrila na solução proporciona um aumento no índice de refração indicando a formação de clusters na solução.
\end{abstract}

\section{INTRODUÇÃO}

O petróleo é hoje a principal matéria-prima de uma vasta quantidade de produtos, entre eles, os plásticos, combustíveis e químicos. Entretanto, as reservas de petróleo são finitas e a sua exploração tem gerado graves problemas ambientais. Dentre as tecnologias que vem sendo desenvolvidas para a substituição do petróleo, a utilização de biomassa como uma fonte de energia vem despertando o interesse de diversos grupos de investigadores (Corma et al., 2007). Estudos apontam o potencial da biomassa como uma fonte alternativa para a produção de produtos químicos e materiais (Haveren et al., 2008). Para a transformação da biomassa, complexos semelhantes a uma refinaria de petróleo tem sido desenvolvidos criando o conceito de biorrefino. Entretanto, a utilização de uma biorrefinaria na conversão de biomassa em produtos de alto valor agregado ainda carece de conhecimento. Dentre os diversos estudos que devem ser realizados, a avaliação de grandezas cinéticas e termodinâmicas é imprescindível para o desenvolvimento de meios reacionais e processos de separação.

O índice de refração $(\eta)$, definido como a razão entre a velocidade da luz no vácuo e a velocidade da luz no meio, pode ser utilizado como uma medida da polarizabilidade eletrônica de uma molécula e pode fornecer informações úteis tanto no estudo das forças intermoleculares presentes em uma solução (Tariq et al., 2009) como na simulação de processos, design de equipamentos e dinâmica molecular (Shukla et al., 2012). A determinação precisa do $\eta$ de um líquido proporcionou um aumento do interesse na sua correlação empírica e teórica com propriedades que são mais difíceis de medir experimentalmente, como por exemplo, a permissividade dielétrica (Brocos et al., 2003). A 
relação entre a polarizabilidade de uma solução e seu índice de refração podem revelar determinadas características estruturais e de ordenamento ao longo de todo o intervalo de composição (Martins et al., 2012).

Apesar da simplicidade experimental envolvida no estudo do $\eta$, a maioria dos trabalhos publicados refere-se a sistemas binários. No entanto, sistemas ternários (ou de ordem superior), não só são mais complexos, mas também potencialmente mais informativos, uma vez que suas propriedades podem ser estudadas através de uma escolha adequada de componentes e composições. Todavia, estudos com sistemas ternários, e principalmente envolvendo sólidos dissolvidos, são escassos na literatura.

Com a finalidade de levantar informações sobre as interações moleculares presentes em uma solução ternária, no presente estudo foi avaliado o comportamento do índice de refração em soluções formadas por água, acetonitrila e glicose, em função da concentração de glicose, concentração de acetonitrila e temperatura da solução. A escolha da glicose na realização do estudo é fundamentada em sua presença na maioria das fontes de biomassa. Água e acetonitrila são componentes frequentemente usados em uma variedade de aplicações como síntese orgânica, cromatografia, hidrometalurgia e eletroquímica (Bakó et al., 2005).

\section{MATERIAL E MÉTODOS}

$\mathrm{Na}$ condução dos experimentos foram utilizadas água bidestilada, acetonitrila P.A e glicose P.A. Todas as quantidades envolvidas foram determinadas por uma balança analítica e colocadas em um balão de fundo chato para facilitar a homogeneização e aguardar até o início do experimento. A solução era então transferida para uma célula de vidro (volume útil de aproximadamente $140 \mathrm{ml}$ ) encamisada com entrada e saída para conexão com o refratômetro óptico (ABBE) e o banho termostático. Para garantir a homogeneização da solução até o final do experimento utilizou-se agitação magnética e para fazer a retirada das amostras utilizou-se uma pipeta adaptada na parte superior da célula de equilíbrio. $O$ monitoramento da temperatura da solução foi feita por um termômetro de bulbo de mercúrio também adaptado na parte superior da célula de equilíbrio.

Após a alimentação da célula com a solução, conexão da célula com o refratômetro e com o banho termostático, regulagem da velocidade de agitação e ajuste da temperatura do banho, o sistema de circulação do fluido de aquecimento era acionado. Durante a primeira hora de experimento a temperatura do banho era ajustada para garantir a precisão da temperatura de ensaio. Com a solução no interior da célula agitada na temperatura de ensaio, o sistema permanecia sob agitação por uma hora e então retirava-se uma amostra da solução e fazia a leitura no refratômetro. A amostragem era feita em triplicata para confirmação da leitura de índice de refração. Feita a leitura do ponto experimental o sistema de aquecimento era ajustado para a próxima temperatura de interesse e o procedimento era repetido. Foram determinados índices de refração para a solução em cinco temperaturas no intervalo de 293,15 $\mathrm{K}$ a $333,15 \mathrm{~K}$.

Inicialmente os dados do índice de refração foram determinados para a água pura e na sequência para as diferentes soluções de água e glicose variando a fração mássica da glicose em $10 \%, 20 \%, 30 \%, 40 \%$ e 50\%. Após realizar o experimento com todas as soluções de água 
e glicose, foi realizado o experimento com soluções, onde além de variar a concentração de glicose também se analisou a influência da acetonitrila em diferentes frações molares até ocorrer à precipitação da glicose. Utilizou-se composições molares de acetonitrila de $10 \%$, $20 \%$ e $30 \%$ em base livre de glicose.

\section{RESULTADOS E DISCUSSÃO}

Os índices de refração determinados para a solução de água e glicose em função da concentração de glicose são apresentados na Tabela 1, para as cinco temperaturas estudadas.

Tabela 1: Índice de refração, com incerteza experimental de $\pm 0,0003$, para a solução binária de água e glicose em diferentes concentrações de glicose e temperaturas

\begin{tabular}{ccccccc}
\hline \multirow{2}{*}{$\mathbf{T}(\mathbf{K})$} & 0 & 10 & 20 & 30 & 40 & 50 \\
\hline 293,15 & 1,3344 & 1,3490 & 1,3655 & 1,3832 & 1,4045 & 1,4276 \\
303,15 & 1,3326 & 1,3481 & 1,3644 & 1,3818 & 1,4036 & 1,4261 \\
313,15 & 1,3315 & 1,3464 & 1,3627 & 1,3802 & 1,4025 & 1,4244 \\
323,15 & 1,3300 & 1,3448 & 1,3614 & 1,3786 & 1,4019 & 1,4235 \\
333,15 & 1,3280 & 1,3434 & 1,3598 & 1,3777 & 1,4003 & 1,4222 \\
\hline
\end{tabular}

Os resultados indicam o aumento do índice de refração com o aumento da concentração de glicose sugerindo a formação de aglomerados de moléculas de glicose que dificultam a passagem de luz no meio, ocasionando assim o aumento do índice de refração. Chen et al. (2012), fizeram uma simulação da dinâmica molecular de soluções aquosas com diferentes concentrações de glicose a fim de investigar a estrutura microscópica destas soluções. Verificou-se que as ligações de hidrogênio presentes na solução podem ocorrer entre moléculas de água, ou entre moléculas de glicose ou ainda entre moléculas de água e glicose. Porém, o número destas ligações varia à medida que ocorre variação da concentração de glicose. A porcentagem de moléculas de água que são ligadas a outras moléculas de água diminui com o aumento da concentração de glicose, bem como as ligações entre moléculas de água e glicose aumentam indicando assim aumento das interações entre a água e glicose. Com o aumento da concentração de glicose, o número médio de moléculas de água por moléculas de glicose diminui, resultando na formação de aglomerados mais complexos das moléculas de glicose.

Ao variar a temperatura em uma mesma concentração de glicose, verifica-se uma diminuição do índice de refração com o aumento da temperatura devido a existência de interações moleculares mais fracas quando se aumenta a temperatura levando a uma diminuição dos aglomerados de glicose e facilitando a passagem de luz no meio.

Na Tabela 2 são apresentados os resultados para o índice de refração das soluções ternárias formadas por água, glicose e acetonitrila em função da temperatura e das concentrações de glicose e acetonitrila. Para a solução com fração molar 0,3 de acetonitrila a concentração de glicose foi variada até $30 \%$ pois com concentração acima observou-se formação de fase sólida a temperatura ambiente. Os resultados para as soluções ternárias indicam um comportamento semelhante ao observado para soluções binárias ou seja, para cada concentração fixa de acetonitrila, verifica-se que com o aumento da temperatura existe 
uma diminuição do índice de refração e com o aumento da concentração de glicose existe um aumento do índice de refração.

Tabela 2: Índice de refração, com incerteza experimental de $\pm 0,0003$, das soluções ternárias de água, glicose e acetonitrila para todas as temperaturas investigadas

\begin{tabular}{ccccccc}
\hline \% Acetonitrila & \% Glicose & $\mathbf{2 9 3 , 1 5 ~ K}$ & $\mathbf{3 0 3 , 1 5 ~ K}$ & $\mathbf{3 1 3 , 1 5} \mathbf{~ K}$ & $\mathbf{3 2 3 , 1 5 ~ K}$ & $\mathbf{3 3 3 , 1 5 ~ K}$ \\
\hline \multirow{4}{*}{10} & 0 & 1,3396 & 1,3377 & 1,3355 & 1,3336 & 1,3311 \\
& 10 & 1,3527 & 1,3508 & 1,3488 & 1,3469 & 1,3450 \\
& 20 & 1,3641 & 1,3625 & 1,3603 & 1,3589 & 1,3571 \\
& 30 & 1,3750 & 1,3730 & 1,3712 & 1,3700 & 1,3680 \\
& 40 & 1,3860 & 1,3834 & 1,3826 & 1,3797 & 1,3790 \\
& 50 & 1,3938 & 1,3905 & 1,3884 & 1,3865 & 1,3836 \\
& 0 & 1,3440 & 1,3410 & 1,3388 & 1,3361 & 1,3330 \\
20 & 10 & 1,3570 & 1,3543 & 1,3522 & 1,3492 & 1,3470 \\
& 20 & 1,3677 & 1,3654 & 1,3632 & 1,3615 & 1,3591 \\
& 30 & 1,3777 & 1,3755 & 1,3738 & 1,3710 & 1,3690 \\
& 40 & 1,3867 & 1,3840 & 1,3816 & 1,3797 & 1,3780 \\
& 50 & 1,3965 & 1,3938 & 1,3912 & 1,3888 & 1,3868 \\
30 & 0 & 1,3461 & 1,3432 & 1,3404 & 1,3373 & 1,3341 \\
& 10 & 1,3585 & 1,3554 & 1,3530 & 1,3505 & 1,3473 \\
& 20 & 1,3690 & 1,3662 & 1,3635 & 1,3612 & 1,3586 \\
& 30 & 1,3807 & 1,3765 & 1,3745 & 1,3715 & 1,3689 \\
\hline
\end{tabular}

Takamuku et al. (2007), estudaram a heterogeneidade de soluções de água e acetonitrila em função da temperatura apontando que nessas soluções as moléculas de água não são homogeneamente misturados com as moléculas de acetonitrila, a nível molecular, mesmo em condições ambientais. $\mathrm{O}$ que ocorre é a formação de duas fases uma rica em acetonitrila e outra rica em água, homogêneas a olho nu, porém heterogênea a nível molecular. Na fase rica em água, uma estrutura tetraédrica das moléculas de água é formada devido às ligações de hidrogênio, e as moléculas de acetonitrila como são excluídas das ligações com a água, são firmemente agregadas pela interação dipolo-dipolo entre elas formando assim clusters de acetonitrila. $\mathrm{Na}$ fase rica em acetonitrila, as moléculas de água podem facilmente serem ligadas entre si pelas ligações de hidrogênio, porque as moléculas de água estão no meio hidrofóbico de acetonitrila, isso pode ser explicado porque as moléculas de água e acetonitrila interagem principalmente por fracas forças dipolo-dipolo e não pelas fortes ligações de hidrogênio como acontece entre as moléculas de água. Com a diminuição da temperatura as interações água-água são reforçadas bem como as interações acetonitrila-acetonitrila tanto na fase rica em água quanto na fase rica em acetonitrila, assim ocorre auto agregação das moléculas de água pelas ligações de hidrogênio e das moléculas de acetonitrila pelas interações dipolo-dipolo.

No presente estudo verifica-se, que quanto menor a concentração de acetonitrila, menores também são os valores de índice de refração, isso pode ser relacionado que quanto menos acetonitrila na solução, menos clusters de acetonitrila-acetonitrila são formados, bem como ocorre pouca interação entre as moléculas de água e acetonitrila e com isso há grande quantidade de água e poucos clusters, facilitando a passagem de luz no meio, ou seja, a 
velocidade da passagem da luz no meio é maior e consequentemente o índice de refração é menor.

Analisando as três concentrações de acetonitrila, e as cinco concentrações de glicose, percebe-se que aumentando a concentração de acetonitrila, e a concentração de glicose em uma mesma temperatura, os valores de índice de refração são aumentados também, ou seja, ocorre uma separação mais distinta da fase rica em água, e a fase rica em acetonitrila, e com isso mais clusters de água na fase rica em água, bem como mais clusters de acetonitrila na fase rica em acetonitrila são formados. Em ambas as fases, como há presença de grande quantidade de moléculas de glicose, estas também interagem mais entre si, do que com as outras moléculas, formando clusters de glicose, tanto na fase rica em água quanto na fase rica em acetonitrila e com isso ocorre um aumento do valor de índice de refração, pois há muitos clusters na solução o que dificulta a passagem de luz do meio. Porém, aumentando a temperatura os valores de índice de refração diminuem, pois a interação entre as moléculas diminui quebrando os clusters e facilitando a passagem de luz no meio.

A partir da medida do índice de refração para todas as soluções nas seis temperaturas estudadas, foi calculado o desvio do índice de refração $(\Delta \eta)$ também conhecido como sinergia do índice de refração ou de sinergia absoluta do índice de refração (Belda et al., 2005), conforme apresentado pela Equação (1) em que $\eta_{\text {exp }}$ representa o valor experimental do índice de refração, $\eta_{i}$ é o valor do índice de refração para os componentes puros e $x_{i}$ é o valor da fração molar do componente na solução.

$\Delta \eta=\eta_{\exp }-\sum_{i=1}^{2} \eta_{i} x_{i}$

Valores positivos de $\Delta \eta$ indicam fracas interações intermoleculares (forças de dispersão) e valores negativos de $\Delta \eta$ são indicativos de fortes interações intermoleculares (Subhash et al., 2009).

Tabela 3: Desvio do índice de refração para as soluções estudadas

\begin{tabular}{ccccccc}
\hline \% Acetonitrila & \% Glicose & $\mathbf{2 9 3 , 1 5 ~ K}$ & $\mathbf{3 0 3 , 1 5} \mathbf{~ K}$ & $\mathbf{3 1 3 , 1 5} \mathbf{~ K}$ & $\mathbf{3 2 3 , 5} \mathbf{~ K}$ & $\mathbf{3 3 3 , 1 5 ~ K}$ \\
\hline \multirow{4}{*}{10} & 0 & 0,00471 & 0,00476 & 0,00384 & 0,00360 & 0,00324 \\
& 10 & 0,00401 & 0,00332 & 0,00328 & 0,00323 & 0,00301 \\
& 20 & 0,00055 & 0,00042 & 0,00024 & 0,00048 & 0,00060 \\
& 30 & $-0,00414$ & $-0,00436$ & $-0,00420$ & $-0,00345$ & $-0,00411$ \\
& 40 & $-0,01178$ & $-0,01302$ & $-0,01228$ & $-0,01409$ & $-0,01281$ \\
& 50 & $-0,02416$ & $-0,02555$ & $-0,02557$ & $-0,02610$ & $-0,02727$ \\
& 0 & 0,00855 & 0,00767 & 0,00695 & 0,00609 & 0,00529 \\
20 & 10 & 0,00865 & 0,00752 & 0,00766 & 0,00680 & 0,00659 \\
& 20 & 0,00625 & 0,00581 & 0,00597 & 0,00628 & 0,00615 \\
& 30 & 0,00276 & 0,00273 & 0,00337 & 0,00288 & 0,00267 \\
& 40 & $-0,00433$ & $-0,00520$ & $-0,00562$ & $-0,00594$ & $-0,00527$ \\
& 50 & $-0,01198$ & $-0,01237$ & $-0,01250$ & $-0,01308$ & $-0,01292$
\end{tabular}


30

\begin{tabular}{ccccccc} 
& 0 & 0,01002 & 0,00943 & 0,00835 & 0,00728 & 0,00656 \\
30 & 0,01054 & 0,00940 & 0,00955 & 0,00951 & 0,00864 \\
& 20 & 0,00975 & 0,00924 & 0,00925 & 0,00935 & 0,00938 \\
& 30 & 0,01001 & 0,00839 & 0,00911 & 0,00879 & 0,00843 \\
\hline
\end{tabular}

Como a glicose apresenta-se como um cristal sólido a temperatura ambiente, a aplicação da Equação (1) é realizada considerando-se a solução ternária como pseudobinária formada por água + glicose (1) e acetonitrila (2). A Tabela 3 mostra os valores de $\Delta \eta$ para as soluções ternárias investigadas. A Figura 1 ilustra o comportamento do desvio do índice de refração para as soluções com fração molar de acetonitrila de 0,1 e 0,2. Os resultados de desvio do índice de refração apresentaram um valor de incerteza experimental média estimada em 6,7.10 $0^{-4}$ aplicando-se o método de propagação de incertezas (PUGH e WINSLOW, 1966), baseado na Equação (2).

$F=F(x, y, \ldots) \rightarrow \delta(F)=\left(\left(\frac{\partial F}{\partial x}\right)^{2} \cdot(\delta x)^{2}+\left(\frac{\partial F}{\partial y}\right)^{2} \cdot(\delta y)^{2}+\cdots\right)^{\frac{1}{2}}$

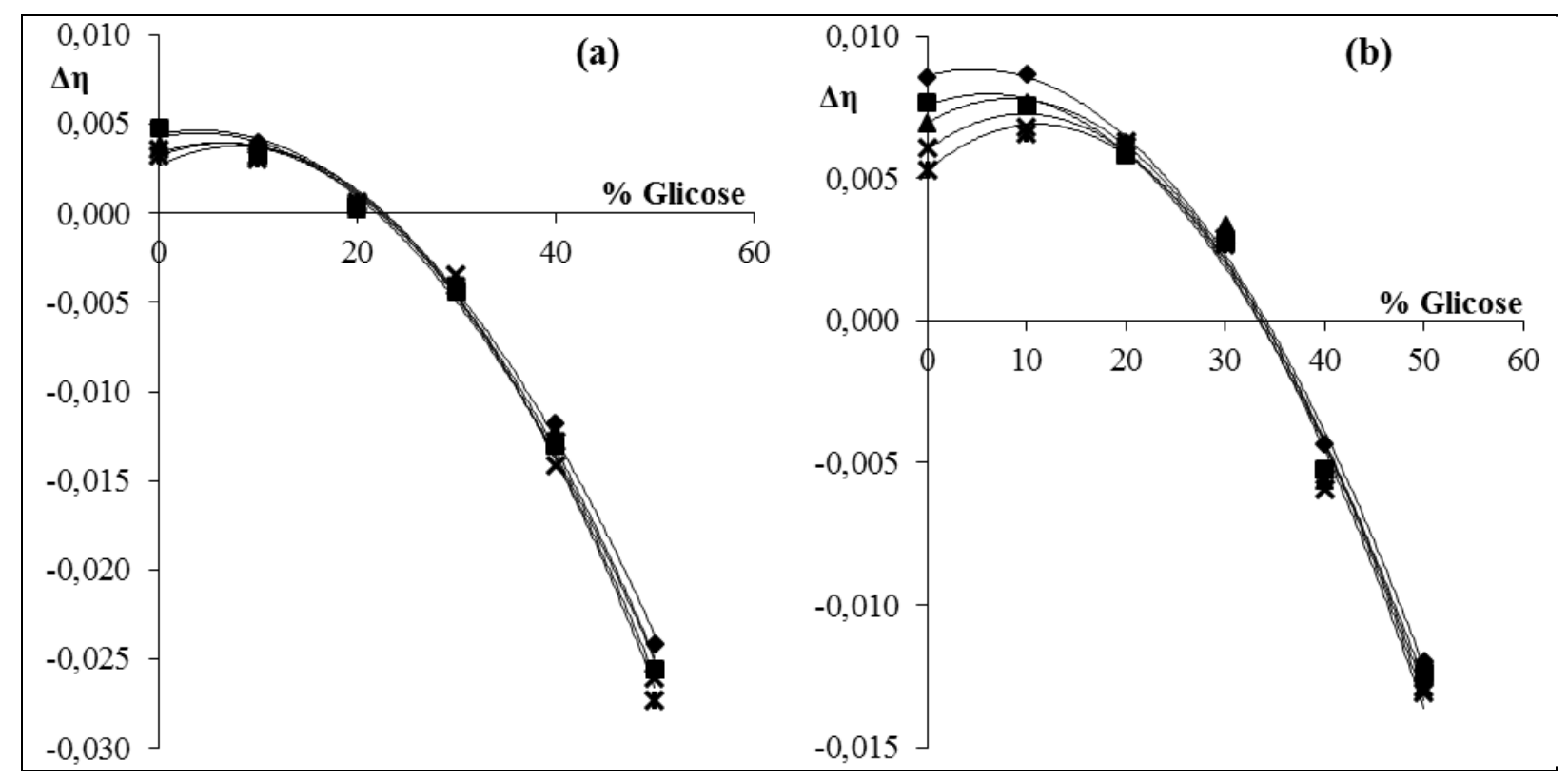

Figura 1: Desvio do índice de refração para as soluções ternárias com fração molar de acetonitrila 0,1 (a) e 0,2 (b) em base livre de glicose: $\bullet 293,15 \mathrm{~K}$; $\boldsymbol{\square} 303,15 \mathrm{~K}$; $\boldsymbol{\Delta}$ 313,15K; $\boldsymbol{x}$ $323,15 \mathrm{~K}$ e $* 333,15 \mathrm{~K}$.

Analisando os resultados apresentados na Tabela 3 observa-se que quanto mais acetonitrila na solução, maior também é o intervalo de valores de $\Delta \eta$ positivos. Este comportamento pode ser explicado devido ao fato que com a adição de acetonitrila as interações entre moléculas diferentes vão se tornando mais fracas, e a interação entre as moléculas iguais mais fortes, resultando na formação de mais clusters. Analisando individualmente a Figura 1, verifica-se que com o aumento da concentração de glicose na 
solução, com concentração fixa de acetonitrila, os valores de $\Delta \eta$ vão diminuindo, indicando que as moléculas de glicose estão interagindo tanto com a água quanto com a acetonitrila, bem como com outras moléculas de glicose, e com isso menos aglomerados só de moléculas de glicose são formados. Isso indica que a presença de moléculas de acetonitrila tem mais influência na formação de clusters do que as moléculas de glicose, ou seja, as moléculas de acetonitrila interagem preferencialmente com outras moléculas de acetonitrila. Aumentando a concentração de acetonitrila na solução há um aumento do índice de refração, este comportamento sugere que como há formação destes clusters de acetonitrila a velocidade de luz no meio é diminuída ocasionando assim o aumento do índice de refração. Na solução com $30 \%$ de acetonitrila, verifica-se que a variação dos valores de $\Delta \eta$ é menor, ou seja, as moléculas de glicose estão interagindo com a água e acetonitrila, porém grande parte ainda prefere a interação com outras moléculas de glicose, formando clusters de glicose, e as moléculas de acetonitrila como interagem fracamente com as moléculas de água, estão interagindo entre elas, resultando em grande número de clusters de acetonitrila.

\section{CONCLUSÃO}

Neste trabalho o índice de refração de soluções binárias formadas por água e glicose, e de soluções ternárias formadas por água, glicose e acetonitrila foi determinado experimentalmente nas temperaturas de $293,15,303,15,313,15,323,15$ e $333,15 \mathrm{~K}$ com a finalidade de observar o comportamento do índice de refração da solução em função da concentração de glicose, concentração de acetonitrila e temperatura. O comportamento observado experimentalmente se apresenta em concordância com resultados de dinâmica molecular apresentado na literatura.

A nível molecular não ocorre mistura do componente água e acetonitrila, e a interação entre moléculas iguais é maior do que entre molecular diferentes, facilitando com isso a formação de aglomerados que dificultam a passagem da luz no meio gerando um aumento nos valores de índice de refração. A presença de moléculas de glicose na solução favorece a interação entre elas, e devido a fortes ligações de hidrogênio ocorre formação de aglomerados. Ao aumentar à temperatura a intensidade das ligações entre as moléculas diminuiu, ocasionando assim uma diminuição dos aglomerados e consequentemente uma diminuição do índice de refração.

Foi calculado o desvio do índice de refração das soluções com o objetivo de verificar a intensidade que ocorre às interações moleculares. Com o aumento da concentração de acetonitrila, os valores de $\Delta \eta$ vão se tornando mais positivos, e analisando somente a variação de glicose os valores de $\Delta \eta$ vão se tornando mais negativos, indicando assim que o reagente acetonitrila não interage fortemente nem com a água nem com a glicose, prefere a interação com outras moléculas de acetonitrila, e com isso há formação de mais clusters de acetonitrila.

\section{REFERÊNCIAS}

BAKÓ, I.; MEGYES, T.; PÁLINKÁS, G. Structural investigation of water-acetonitrile mixtures: An ab initio, molecular dynamics and X-ray diffraction study. Chem. Phys., v. 316, p.235-244, 2005. 
BELDA, R.; HERRÁEZ J. V.; DIEZ, O. A study of the refractive index and surface tension synergy of the binary water/ethanol: influence of concentration. Phys. Chem. Liq., v. 43, p.91-101, 2005.

BROCOS, P.; PIÑEIRO, A.; BRAVO, R.; AMIGO, A. Refractive indices, molar volumes and molar refractions of binary liquid mixtures: concepts and correlations. Phys. Chem. Chem. Phys., v. 5, p.550-557, 2003.

CHEN, C.; LI, W.Z.; SONG, Y.C.; WENG, L.D.; ZHANG, N. Formation of water and glucose clusters by hydrogen bonds in glucose aqueous solutions. Comp. Theor. Chem., v. 984, p.85-92, 2012.

CORMA, A.; IBORRA, S.; VELTY, A. Chemical routes for the transformation of biomass into chemicals. Chem. Rev., v. 107, p.2411-2502, 2007.

HAVEREN, J.V.; SCOTT, E.L.; SANDERS, J. Bulk chemicals from biomass. Biofuels, Bioprod. Bior., v. 2, p.41-57, 2008.

MARTINS, F.; LEITÃO, R.E.; VENTURA, M.C.; PINHEIRO, L.; NUNES, N. Densities and refractive indices for the ternary mixture methanol/propan-1-ol/acetonitrile. J. Mol. Liq., v. 170, p.30-36, 2012.

PUGH, E.M; WINSLOW, G.H. The analysis of physical measurements, Addison-Wesley, Reading, Mass., 1966.

SHUKLA, R.K.; KUMAR, A. AWASTHI, N.; SRIVASTAVA, U. GANGWAR, V.S. Density, viscosity and refractive index of binary liquid mixtures at 293.15, 298.15, 303.15, 308.15 and 313.15 K. Exp. Therm. Fluid Sci., v. 37, p.1-11, 2012.

SUBHASH, C.B.; BHATIA, R.; DUBEY, G.P. Refractive properties and internal pressures of binary mixtures of octan-1-ol with chloroform, 1,2-dichloroethane and 1,1,2,2tetrachloroethane at 298.15 and 308.15 K. J. Mol. Liq., v. 145, p.88-102, 2009.

TAKAMUKU, T.; NOGUCHI, Y.; MATSUGAMI, M.; IWASE, H.; OTOMO, T.; NAGAO, M. Heterogeneity of acetonitrile-water mixtures in the temperature range 279-307 K studied by small-angle neutron scattering technique. J. Mol. Liq., v. 136, p.147-155, 2007.

TARIQ, M.; FORTE, P.A.S.; GOMES, M.F.C.; LOPES, J.N.C.; REBELO, L.P.N.Densities and refractive indices of imidazolium- and phosphonium-based ionic liquids: Effect of temperature, alkyl chain length, and anion. J. Chem. Thermodyn., v. 41, p.790-798, 2009. 